\title{
Recueil de l'ensemble des textes d'information destinés aux professionnels de la santé
}

\section{Karoline Mathys}

Karoline Mathys dirige le Secteur Surveillance du marché et est membre de la Direction de Swissmedic.
Correspondance: Swissmedic

Institut suisse des produits thérapeutiques

Hallerstrasse 7

Case postale

CH-3000 Berne 9

Tél. 0313220211

www.swissmedic.ch
Depuis janvier 2013, la nouvelle plate-forme en ligne AIPS (système de publication des textes d'information sur les médicaments de Swissmedic), sur laquelle sont publiées l'ensemble des informations destinées aux patients et aux professionnels de la santé à propos de tous les médicaments autorisés, est disponible sur www.swissmedicinfo.ch ou par le biais du site Internet de Swissmedic. Les médecins et les collaborateurs dans le secteur des soins de santé ont ainsi un accès garanti à un outil rassemblant toutes les informations nécessaires pour utiliser les médicaments correctement et en toute sécurité.

En juin 2011, le Tribunal administratif fédéral a rendu une décision selon laquelle Swissmedic ne pouvait pas contraindre les entreprises à faire publier à leurs frais les textes de l'information professionnelle et de l'information destinée aux patients par un éditeur privé. Cette décision a conduit à un éparpillement des informations concernant les médicaments sur différentes plate-formes: au lieu d'opter pour un recueil qui vise l'exhaustivité comme le Compendium des médicaments, certaines entreprises ont fait publier ces textes par différents fournisseurs, ou les ont mis à disposition exclusivement sur leur propre site Internet.

La plate-forme en ligne de Swissmedic: un outil au service de la sécurité des médicaments

Les informations professionnelles et les informations destinées aux patients sur les différents médicaments sont adaptées en permanence aux dernières avancées scientifiques - un tiers de ces textes environ sont modifiés chaque année. Or si l'on souhaite garantir la sécurité des médicaments, il est impératif que tant les professionnels de la santé que le public aient accès à un recueil électronique exhaustif et à jour rassemblant les informations relatives à tous les médicaments autorisés en Suisse. Telle est la raison pour laquelle l'institut a décidé de mettre lui-même à disposition une plate-forme de publication, même s'il n'est pas obligé de le faire d'un point de vue juridique. Cependant, Swissmedic se cantonne à fournir gracieusement l'environnement correspondant et n'alimente donc pas cette plate-forme du point de vue du contenu. C'est donc toujours aux titulaires des autorisations qu'incombe la responsabilité de veiller à ce que les dernières versions des informations sur les médicaments soient toujours en ligne, et de s'assurer que les traductions soient correctes. Afin que les professionnels de la santé soient mieux informés au sujet des dernières découvertes pertinentes pour la sécurité, les préparations qui ont fait l'objet d'une publication correspondante (HPC) dans les 12 mois précédents sont mises en exergue et pourvues d'un lien vers l'aperçu disponible sur le site Internet de Swissmedic. L'on peut également obtenir une liste de tous les textes modifiés pendant les quatre semaines précédentes. Pendant la phase de mise en place jusqu'à mi-2013, cette liste reprendra non seulement les textes dont la teneur a été modifiée mais également ceux dont une nouvelle version a été mise en ligne suite à des modifications formelles.

\section{Une plate-forme, plusieurs applications}

La plate-forme AIPS se compose d'un moteur de recherche en libre accès (système d'information), et d'une partie protégée destinée à la mise en ligne et au remaniement des textes par les titulaires d'autorisations (système de publication). Nous précisons que ce recueil ne fournit que les données prévues par la législation sur les produits thérapeutiques (informations professionnelles et informations destinées aux patients qui ont été approuvées par Swissmedic) et qu'il n'apporte donc aucun autre renseignement (tarifs ou données relevant de l'assurance-maladie par exemple). D'autres fournisseurs de recueils électroniques d'informations sur les produits thérapeutiques peuvent toutefois utiliser librement ces données de base et les intégrer sans entrave technique dans leurs propres recueils au format XML. Il peuvent ainsi compléter ces données avec des informations complémentaires, ou les mettre à disposition par d'autres canaux (ebooks, apps, documents imprimés, transfert vers des systèmes d'information électroniques internes).

Le 31 décembre 2012, les textes publiés jusqu' alors en format électronique dans le Compendium des médicaments ont été transférés vers la nouvelle banque de données AIPS. Dès à présent, les titulaires d'autorisations sont invités à vérifier si les informations sur les médicaments publiées sont à jour et à ajouter les textes manquants sur la nouvelle plateforme de Swissmedic. Nous soulignons par ailleurs que désormais, les traductions italiennes des informations destinés aux patients devraient également être disponibles. L'objectif de ce projet est de mettre à disposition pour mi-2013 un recueil rassemblant tous les textes d'information destinés aux professionnels de la santé et aux patients. 